\title{
Full-duplex decode-and-forward relaying with joint relay-antenna selection
}

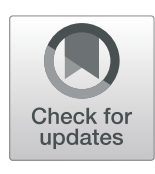

Mahsa Shirzadian Gilan ${ }^{1,2+}$ and Ha H. Nguyen ${ }^{2 *+}$ (D)

\begin{abstract}
This paper is concerned with wireless relay networks that employ $K$ full-duplex (FD) decode-and-forward relays to help a source to communicate with a destination. Each FD relay is equipped with multiple antennas, some for receiving and some for transmitting. The paper considers joint relay-antenna selection schemes that are based on the instantaneous channel conditions for two cases of antenna configurations, namely fixed antenna configuration (FAC) and adaptive antenna configuration (AAC). Under FAC, the transmit and receive antennas at each relay are fixed, whereas in the case of AAC an antenna at a relay can be configured to be either a transmit or a receive antenna. In addition to equal power allocation between the source and selected relay, a power scaling approach to counteract the effect of residual self-interference is also examined. Closed-form expressions of the outage probability and average capacity are obtained and provide important insights on the system performance. The accuracy of the obtained expressions are corroborated by simulation results. In particular, it is shown that under FAC and without power scaling, the diversity order approaches $K$ as the self-interference (SI) level gets smaller, while it approaches zero whenever the $\mathrm{SI}$ level is nonzero and the SNR increases without bound. Under FAC and with power scaling, the diversity order approaches $K$ for any $S I$ level. For the case of AAC and without power scaling, the diversity order approaches $2 K$ for small SI level. When power scaling is applied in AAC, the diversity order approaches $2 K$ at any SI level.
\end{abstract}

Keywords: Full-duplex, Antenna mode selection, Relay selection, Self-loop interference, DF relaying

\section{Introduction}

Full-duplex (FD) communications allow simultaneous transmission and reception on the same frequency band, which theoretically achieves twice the spectral efficiency as compared to half-duplex (HD) communications [1]. A critical issue in FD communications is that the selfinterfering signal from the FD transmitter is much stronger than the intended receiving signal. Thanks to advanced self-interference cancellation techniques developed in recent years, both in analog and digital signal processing, FD communications have been introduced in recent fifth-generation (5G) standard proposals as an appealing technique to significantly enhance the attainable spectral efficiency of communication systems [1-3].

Given that traditional HD relaying has been shown to greatly extend the coverage and/or significantly reduce

\footnotetext{
*Correspondence: ha.nguyen@usask.ca

${ }^{\dagger}$ Mahsa Shirzadian Gilan and Ha H. Nguyen contributed equally to this work.

${ }^{2}$ Department of Electrical and Computer Engineering, University of

Saskatchewan, Saskatoon, Canada

Full list of author information is available at the end of the article
}

power consumption in wireless networks, it is natural to consider FD communications in the context of wireless relay networks [4]. Although various self-interference (SI) cancellation schemes have been developed [5-7], residual self-interference always remains in practice due to imperfect cancellation. The residual interference is modeled as a Rayleigh distributed random variable in [8] and the outage performance of dual-hop FD relaying was analyzed accordingly. Reference [9] further extends [8] to a multihop FD relay system and takes into account the path loss factor. The authors show that, with effective selfinterference cancellation, FD relaying outperforms HD relaying. To deal with severe self-interference, a sophisticated hybrid FD/HD relaying scheme was studied in [10] which adaptively switches between FD and HD modes based on the instantaneous SI level. Various FD relaying schemes were investigated in [11] that are based on the codeword expansion technique. Such a technique benefits from time diversity that is dependent on the efficiency of the SI cancellation. The authors in [12] consider an optimization problem to find the power and location

\section{Springer Open}


of relays so that the effect of self-interference can be minimized. Performance of MIMO FD relaying in the presence of co-channel interference is analyzed in [13], in which closed-form expressions for outage probability and ergodic capacity are derived. Several antenna selection schemes have been investigated in $[14,15]$ to maximize the end-to-end performance of the multiple-antenna amplify-and-forward (AF) relay systems. The authors in [16] examines a FD two-way relay network that is made up of one base station (BS), one FD AF relay, and one user, and the BS is equipped with massive antennas (massive MIMO). To reduce complexity and cost of the BS, the authors propose a practical antenna selection scheme at the BS. They obtain closed-form expressions for the outage probability and average BER under Nakagami- $m$ fading channels and demonstrate performance superiority of their proposed antenna selection scheme over the conventional scheme.

When multiple FD relays are employed, relay selection is an efficient and simple approach to achieve the spatial diversity as compared to other alternatives, such as distributed space-time coding. In [17], several relay selection schemes were proposed to optimize the end-toend signal-to-interference-plus-noise ratio (SINR) in AF cooperative FD relay networks that take into account the residual self-interference. By analyzing the outage probability, the authors show that, although the effect of the self-interference is reduced, the residual self-interference is the main drawback of a FD relay system.

All the works on FD relaying systems discussed above assume that the roles of the transmit and receive antennas are unchanged in the relaying process. When the channel link from the source to the relay's receive antenna and/or from the relay's transmit antenna to the destination is in deep fading, the system performance will be seriously degraded. Based on this observation the authors in [18] consider a FD relaying system in which the antennas of each FD relay can be configured to transmit (Tx) or receive $(\mathrm{Rx})$ the signal. In particular, the authors propose a joint relay and $T x / R x$ antenna mode selection scheme (RAMS), where the optimal relay with its optimal $\mathrm{Tx} / \mathrm{Rx}$ antenna configuration is selected jointly based on the instantaneous channel conditions. Only the optimal relay is active to forward the information from the source to the destination using the AF protocol. In doing so, the proposed scheme provides an additional dimension of selection process, which introduces an extra degree of freedom compared to the conventional FD relay selection. The authors in [18] obtain the CDF of the end-to-end SINR for their proposed RAMS scheme, as well as closedform expressions of the outage probability and ergodic capacity. In addition, they also propose an adaptive power allocation to mitigate the self-interference and reduce the error floor.
Regarding relay networks that employ multiple FD relays and the decode-and-forward (DF) protocol, the authors in [19] analyze the outage performance over Nakagami- $m$ fading channels. More recently, performance of the FD system with DF relay selection is analyzed in [20], in which the authors demonstrate that the error floor in the high SNR regime can be mitigated. However, the scheme considered in [20] requires decoding at all relays and knowing whether the decoded symbols at relays are correct. This implicitly assumes decoding to bits and powerful CRC codes are used, which leads to higher complexity as well as reduced bandwidth efficiency.

From the above discussion and particularly motivated by the work in [20], this paper considers a FD relay system that employs multiple FD relays and the DF protocol. As in [20], it is assumed that each FD relay is equipped with multiple antennas and two cases are examined. In the first case, referred to as fixed antenna configuration (FAC), the transmit and receive antennas at each relay are fixed. On the other hand, in the second case, called adaptive antenna configuration (AAC), an antenna at a relay can be configured to be either a transmit or a receive antenna, which implies that there are flexible connection switches between the antennas and the RF chains [20]. In either case, joint relay-antenna selection is performed based on the instantaneous channel conditions so that the minimum SINR via any relying link is maximized. By taking into account the residual self-interference, outage performance of the considered joint relay-antenna selection is derived and ergodic capacity results are obtained. Furthermore, a power scaling approach is investigated to mitigate the outage error floor at the high SNR regime.

It is pointed out that, in addition to consider the integration of FD communications with relaying technology and joint antenna-relay selection, it would be very interesting to also integrate energy harvesting into the system model considered in this paper. Indeed, energy-harvesting technology has been extensively studied in various communication systems, including relay-antenna selection in cooperative MIMO/NOMA networks [21], simultaneous wireless information and power transfer in dual-hop relaying networks [22], and UAV relay-assisted IoT networks ${ }^{1}$ [23].

The remainder of the paper is organized as follows. Section 2 describes the methods used in the paper. Section 3 introduces the system model. Sections 4 and 5 present performance analysis of the FAC scheme without and with power scaling, respectively. Section 6 investigates performance of the AAC scheme. Section 7 provides numerical results. Finally, section 8 concludes the paper.

\footnotetext{
${ }^{1}$ The abbreviations of NOMA, UAV, and IoT stand for "non-orthogonal multiple-access", "unmanned aerial vehicle", and "Internet of things", respectively.
} 


\section{Methods}

The research methodology in this paper involves system modeling, theoretical analysis, and computer simulation. System modeling uses tools and insights from information and communication theories to develop simplified but meaningful mathematical models of the design problems. Theoretical analysis is carried out to provide valuable insights into possible design choices and intuitive understanding of the impacts of different design parameters on the system/network performance. To corroborate the theoretical results, simulation models are developed and implemented.

\section{System model}

Figure 1 illustrates the FD relaying system considered in this paper. This system model is similar to that studied in [18] but with a major difference that DF relays are employed instead of AF relays. Here, $K$ FD relays assist one source $(S)$ to communicate with one destination (D). It is assumed that, due to blockage and/or large distance separation between $\mathrm{S}$ and $\mathrm{D}$, the direct link between them is not available. Each relay is equipped with $Q$ antennas, among which $M$ are designated as receive antennas and the remaining $L=Q-M$ are transmit antennas. On the other hand, both source and destination are equipped with a single antenna. Such an assumption is applicable for scenarios when there are size, power and/or cost constraints to put multiple antennas on informationexchange devices (source and destination), while there are no such constraints (or much more relaxed) for the relay.
The channel coefficients from $\mathrm{S}$ to antenna $m(m=$ $1,2, \ldots, M)$ of relay $i(i=1,2, \ldots, K), R_{i}$, and from antenna $l(l=1,2, \ldots, L)$ of $R_{i}$ to $\mathrm{D}$ are denoted by $h_{\mathrm{S}, i}^{(m)}$ and $h_{i, \mathrm{D}}^{(l)}$, respectively. Moreover, the effect of residual self-interference (RSI) at relay $R_{i}$ is represented using the residual self-interference channel $I_{i, i}^{l \rightarrow m}$. All wireless channels are subjected to flat fading and additive white Gaussian noise (AWGN). As in [18], all links are assumed to be independent and characterized with Rayleigh fading. As a result, the squared amplitudes of channel fading coefficients are exponentially distributed.

With the considered joint relay-antenna selection, suppose that relay $R_{i}$ with antenna $m$ for receiving and antenna $l$ for transmitting is selected to assist data transmission from $\mathrm{S}$ to $\mathrm{D}$. At time $n$, the source broadcasts its unit power symbol $x(n)$ to all the relays. The received signal at $R_{i}$ with receive antenna $m$ and transmit antenna $l$ can be written as

$$
y_{\mathrm{S}, i}^{l \rightarrow m}=\sqrt{P_{\mathrm{S}}} x(n) h_{\mathrm{S}, i}^{(m)}+\sqrt{P_{\mathrm{R}}} \hat{x}\left(n-n_{0}\right) I_{i, i}^{l \rightarrow m}+w_{i}(n),
$$

where $P_{S}$ is the transmitted power at the source, $\hat{x}\left(n-n_{0}\right)$ is the decoded symbol at relay $i$ and $n_{0}$ indicates the processing delay at $R_{i}$, and $w_{i}(n)$ is an AWGN sample with zero mean and unit variance. In this paper, the information symbols belong to PSK constellation $\Psi$, i.e., $x(n) \in \Psi$, where $\Psi \equiv\left\{\exp \left(j \frac{\pi(2 k+1)}{|\Psi|}\right), k=0, \ldots,|\Psi|-1\right\}$ and $|\Psi|$ is the cardinality of $\Psi$. Therefore, the received SNR at each

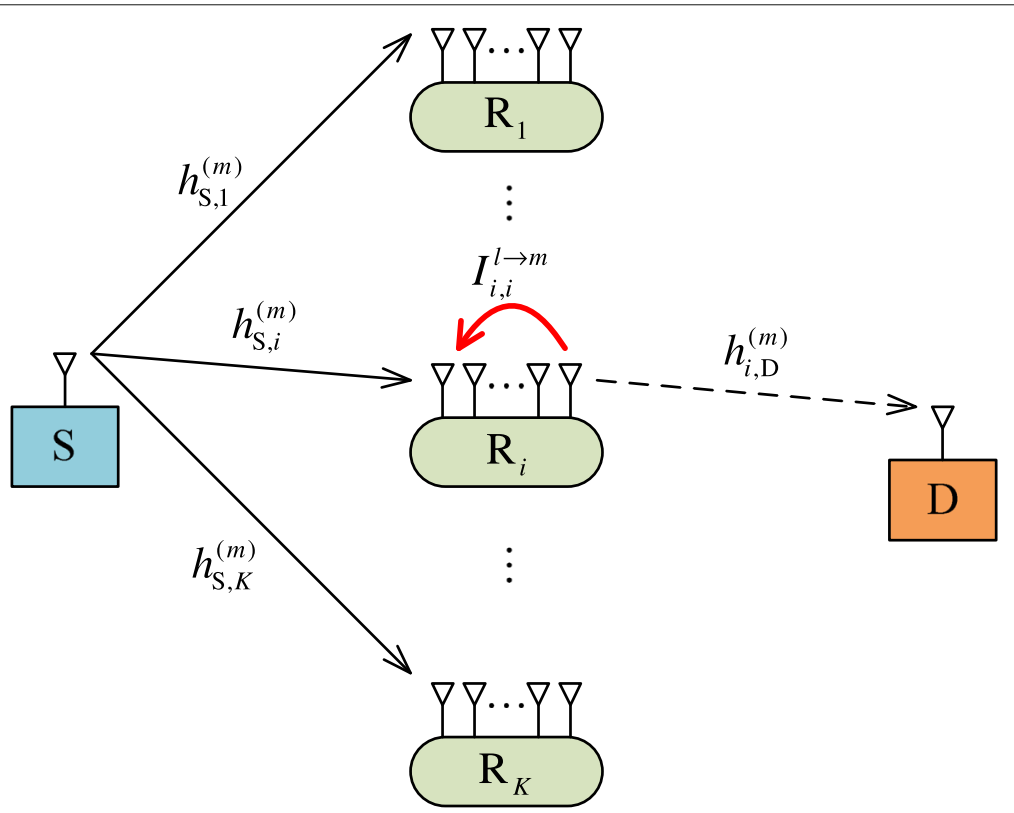

Fig. 1 System model 
FD relay operating on receive antenna $m$ and transmit antenna $l$ can be written as

$$
\gamma_{\mathrm{S}, i}^{l \rightarrow m}=\frac{P_{\mathrm{S}}\left|h_{\mathrm{S}, i}^{(m)}\right|^{2}}{P_{\mathrm{R}}\left|I_{i, i}^{l \rightarrow m}\right|^{2}+1} .
$$

The received signal at the destination is

$$
y_{i, \mathrm{D}}^{l \rightarrow m}=\sqrt{P_{\mathrm{R}}} \hat{x}\left(n-n_{0}\right) h_{i, \mathrm{D}}^{(l)}+w_{\mathrm{D}}(n),
$$

where $P_{\mathrm{R}}$ is the transmitted power at the selected relay and $w_{\mathrm{D}}(n)$ is an AWGN sample with zero mean and unit variance. With PSK constellation, the received SNR at the destination is

$$
\gamma_{i, \mathrm{D}}^{l \rightarrow m}=P_{\mathrm{R}}\left|h_{i, \mathrm{D}}^{(l)}\right|^{2}
$$

Under Rayleigh fading, the squared magnitudes $\left|h_{\mathrm{S}, i}^{(m)}\right|^{2}$, $\left|h_{i, \mathrm{D}}^{(l)}\right|^{2}$, and $\left|I_{i, i}^{l \rightarrow m}\right|^{2}$ are exponentially distributed random variables with parameters $\lambda_{\mathrm{S}, i}, \lambda_{i, \mathrm{D}}$, and $\lambda_{i, i}$, respectively. This means that the average power gains of these channels are $1 / \lambda_{\mathrm{S}, i}, 1 / \lambda_{i, \mathrm{D}}$, and $1 / \lambda_{i, i}$ and they do not depend on the particular pair $(l, m)$ of receive/transmit antennas at the selected relay.

This paper considers joint relay-antenna selection in order to maximize the minimum SINR via any relaying link connecting $S$ and $D$. In particular, the joint relayantenna selection schemes studied for the two cases of antenna configurations are as follows:

- Fixed antenna configuration (FAC): In this case, the sets of $M \mathrm{Rx}$ and $L \mathrm{Tx}$ antennas are fixed. The considered joint relay-antenna selection is as follows:

$$
\{i, l, m\}=\arg \max _{i} \max _{\{l, m\}} \min \left\{\gamma_{S, i}^{l \rightarrow m}, \gamma_{i, D}^{l \rightarrow m}\right\}
$$

Note that the number of all states for $i, l, m$ is KLM.

- Adaptive antenna configuration (AAC): In this case the sets of Rx and Tx antennas are not fixed, but the optimal $R x$ and Tx antennas are selected jointly based on the instantaneous channel conditions. This means that each antenna of the FD relay is able to transmit or receive the signal and the relay node has flexible connection switches between the antennas and two RF chains (one for transmitting and one for receiving). The joint relay-antenna selection is performed as follows:

$$
\begin{gathered}
\left\{i, k, l_{k}, m_{k}\right\}=\arg \operatorname{maxmaxmax}_{k} l_{l_{k}, m_{k}}\{ \\
\left.\min \left\{\gamma_{\mathrm{S}, i}^{l_{k} \rightarrow m_{k}}, \gamma_{i, \mathrm{D}}^{l_{k} \rightarrow m_{k}}\right\}, \min \left\{\gamma_{\mathrm{S}, i}^{m_{k} \rightarrow l_{k}}, \gamma_{i, \mathrm{D}}^{m_{k} \rightarrow l_{k}}\right\}\right\}, \\
k \in \mathcal{D}=\left\{1,2, \ldots,\left(\begin{array}{l}
Q \\
2
\end{array}\right)\right\},
\end{gathered}
$$

where $\mathcal{D}$ is the set that contains all permutations to select two antennas among $Q$ antennas at each FD relay. For permutation $k$, also called mode $k, l_{k}$ and $m_{k}$ denote the indices of the transmitting and receiving antennas at each FD relay. The size of $\mathcal{D}$ is $\left(\begin{array}{l}Q \\ 2\end{array}\right)=\frac{Q(Q-1)}{2}$. Because there are two ways to use a pair of antennas for transmitting and receiving, the number of all states for max-min function $(i, k)$ is $K Q(Q-1)$.

\section{Performance analysis under FAC and without power scaling}

Let $\gamma_{i}=\min \left\{\gamma_{\mathrm{S}, i}^{l \rightarrow m}, \gamma_{i, \mathrm{D}}^{l \rightarrow m}\right\}$ be the minimum SINR of the $i$ th relay link. Then the CDF of $\gamma_{i}$ is calculated as

$$
\begin{aligned}
F_{\gamma_{i}}(x) & =\operatorname{Pr}\left\{\gamma_{i}<x\right\}=1-\operatorname{Pr}\left\{\gamma_{i}>x\right\} \\
& =1-\operatorname{Pr}\left\{\gamma_{\mathrm{S}, i}^{l \rightarrow m}>x\right\} \operatorname{Pr}\left(\gamma_{i, \mathrm{D}}^{l \rightarrow m}>x\right) .
\end{aligned}
$$

By substituting the following expressions

$$
\begin{gathered}
\operatorname{Pr}\left\{\gamma_{\mathrm{S}, i}^{l \rightarrow m}>x\right\}=\exp \left(-\frac{x \lambda_{\mathrm{S}, i}}{P_{\mathrm{S}}}\right)\left(1+\frac{x P_{\mathrm{R}} \lambda_{\mathrm{S}, i}}{P_{\mathrm{S}} \lambda_{i, i}}\right)^{-1} \\
\operatorname{Pr}\left\{\gamma_{i, \mathrm{D}}^{l \rightarrow m}>x\right\}=\exp \left(-\frac{\lambda_{i, \mathrm{D}}}{P_{\mathrm{R}}} x\right)
\end{gathered}
$$

into (7), one has

$$
F_{\gamma_{i}}(x)=1-\frac{\exp \left(-\left(\frac{\lambda_{i, \mathrm{D}}}{P_{\mathrm{R}}}+\frac{\lambda_{\mathrm{S}, i}}{P_{\mathrm{S}}}\right) x\right)}{1+x \eta_{i}},
$$

where

$$
\eta_{i}=\frac{\lambda_{\mathrm{S}, i} P_{\mathrm{R}}}{\lambda_{i, i} P_{\mathrm{S}}}=\frac{P_{\mathrm{R}} / \lambda_{i, i}}{P_{\mathrm{S}} / \lambda_{\mathrm{S}, i}}
$$

The parameter $\eta_{i}$ quantifies the amount of residual selfinterference power as a percentage of the power received from the source at the selected relay. For example, if $\eta_{i}=$ 0.01 , then the residual self-interference power is $1 \%$ of the power received from the source.

In the high transmit power regime, $P_{\mathrm{S}}$ and $P_{\mathrm{R}}$ approach infinity, hence $F_{\gamma_{i}}(x)$ in (10) approaches

$$
F_{\gamma_{i}}^{(\infty)}(x)=1-\frac{1}{1+x \eta_{i}} .
$$

The above expression shows that, in the high SNR regime, the distribution of the minimum SINR for any relay link only depends on the self-interference level at the relay node. This means that, in the high SNR regime, increasing the transmitted powers of the source and selected relay is ineffective to enhance the system performance.

\subsection{Outage probability}

In the joint relay-antenna selection scheme considered in this paper, the combination of relay and $\mathrm{Rx} / \mathrm{Tx}$ antennas that yields the largest minimum SINR is selected for relaying information from $S$ to $D$. Therefore, the outage 
probability of the network with $K$ relays can be calculated as

$$
\begin{gathered}
P_{\text {out }}(x)=\operatorname{Pr}\left(\max \left\{\gamma_{1}, \ldots, \gamma_{K}\right\}<x\right)=\prod_{i=1}^{K} \operatorname{Pr}\left\{\gamma_{i}<x\right\} \\
=\prod_{i=1}^{K}\left(1-\frac{\exp \left(-\left(\frac{\lambda_{i, \mathrm{D}}}{P_{\mathrm{D}}}+\frac{\lambda_{\mathrm{S}, i}}{P_{\mathrm{S}}}\right) x\right)}{1+x \eta_{i}}\right)
\end{gathered}
$$

Consider equal transmitted power at the source and selected relay and define the average SNR as $\bar{\gamma}=\frac{P_{\mathrm{S}}}{\sigma_{w}^{2}}=$ $\frac{P_{\mathrm{R}}}{\sigma_{w}^{2}}=P_{\mathrm{R}}$ where $\sigma_{w}^{2}=1$ is the variance of AWGN noise. The finite-SNR diversity order is defined as $[18,24]$

$$
d(\bar{\gamma})=-\frac{\partial \ln P_{\text {out }}(\bar{\gamma})}{\partial \ln \bar{\gamma}},
$$

where $P_{\text {out }}(\bar{\gamma})$ is the outage probability of the FD relaying system at SNR $\bar{\gamma}$.

Given the expression of the outage probability in (13) and applying the recursive rule, the finite-SNR diversity order of the considered system can be shown to be

$$
d=\sum_{i=1}^{K} \frac{x}{P_{\mathrm{R}}}\left(\lambda_{i, \mathrm{D}}+\lambda_{\mathrm{S}, i}\right) \frac{\exp \left(-\left(\frac{\lambda_{i, \mathrm{D}}}{P_{\mathrm{R}}}+\frac{\lambda_{\mathrm{S}, i}}{P_{\mathrm{S}}}\right) x\right)}{1+x \eta_{i}-\exp \left(-\left(\frac{\lambda_{i, \mathrm{D}}}{P_{\mathrm{R}}}+\frac{\lambda_{\mathrm{S}, i}}{P_{\mathrm{S}}}\right) x\right)} .
$$

Furthermore, using Taylor series expansions, (15) can be approximated as

$$
d \approx \sum_{i=1}^{K} \frac{1}{1+\kappa_{i}}
$$

where $\kappa_{i}=\frac{P_{\mathrm{R}} \eta_{i}}{\lambda_{i, \mathrm{D}}+\lambda_{\mathrm{S}, i}}$.

In the case of small self-interference level, one has $\eta_{i} \rightarrow$ 0 and $d \rightarrow K$. Furthermore, as long as the residual self interference level is nonzero, i.e., $\eta_{i} \neq 0$, then in the high SNR regime, $P_{\mathrm{R}} \rightarrow \infty$ and $\kappa_{i} \rightarrow \infty$. This leads to a diversity order of zero, which is a direct consequence of the irreducible floor of the outage probability caused by the self-interference at the FD relay.

\subsection{Average capacity}

The outage probability in (13) can be rewritten as [18]:

$$
P_{\text {out }}(x)=\sum_{\mathcal{A} \subset \mathcal{S}} \prod_{i \in \mathcal{A}} \frac{-\exp \left(-\left(\frac{\lambda_{i, \mathrm{D}}}{P_{\mathrm{R}}}+\frac{\lambda_{\mathrm{S}, i}}{P_{\mathrm{S}}}\right) x\right)}{1+x \eta_{i}} .
$$

In the above expression, $\mathcal{S}=\{1,2, \ldots, K\}, \mathcal{A}$ denotes a subset of $\mathcal{S}$ and the summation is over all possible subsets of $\mathcal{S}$. Therefore, the ergodic capacity can be calculated as [18]

$$
\begin{aligned}
\bar{C} & =\frac{1}{\ln 2} \int_{0}^{\infty} \frac{1-P_{\text {out }}(x)}{1+x} d x \\
& =\sum_{\substack{\mathcal{A} \subset \mathcal{S} \\
\mathcal{A} \neq \emptyset}} \frac{-(-1)^{|\mathcal{A}|}}{\ln 2} \int_{0}^{\infty} \frac{1}{1+x} \prod_{i \in \mathcal{A}} \frac{\exp \left(-\left(\frac{\lambda_{i, \mathrm{D}}}{P_{\mathrm{R}}}+\frac{\lambda_{\mathrm{S}, i}}{P_{\mathrm{S}}}\right) x\right)}{1+\eta_{i} x} d x \\
& =\sum_{\substack{\mathcal{A} \subset \mathcal{S} \\
\mathcal{A} \neq \emptyset}} \frac{-(-1)^{|\mathcal{A}|}}{\ln 2} \int_{0}^{\infty} T(x) \exp \left(-\sum_{i \in \mathcal{A}}\left(\frac{\lambda_{i, \mathrm{D}}}{P_{\mathrm{R}}}+\frac{\lambda_{\mathrm{S}, i}}{P_{\mathrm{S}}}\right) x\right) d x,
\end{aligned}
$$

where $|\mathcal{A}|$ denotes the cardinality of set $\mathcal{A}$ and

$$
T(x)=\frac{1}{(1+x) \prod_{i \in \mathcal{A}}\left(1+\eta_{i} x\right)} .
$$

Using the residue theorem, the function $T(x)$ can be written as

$$
T(x)=\frac{a}{1+x}+\sum_{i \in \mathcal{A}} \frac{b_{i}}{1+\eta_{i} x},
$$

where $a$ and $b_{i}$ are given by

$a=\frac{1}{\prod_{i \in \mathcal{A}}\left(1-\eta_{i}\right)}, \quad b_{i}=\frac{1}{\left(1-\frac{1}{\eta_{i}}\right) \prod_{i^{\prime} \in \mathcal{A}, i^{\prime} \neq i}\left(1-\frac{\eta_{i^{\prime}}}{\eta_{i}}\right)}$.

By using the following identity [25],

$\int_{0}^{\infty} \frac{\mathrm{e}^{-\mu x}}{(x+v)^{n}} d x=\frac{1}{(n-1) !} \sum_{k=1}^{n-1}(k-1) !(-\mu)^{n-k-1} v^{-k}-$ $\frac{(-\mu)^{n-1}}{(n-1) !} \mathrm{e}^{\nu \mu} E_{i}(-v \mu), n \geq 2,|\arg (\nu)|<\pi, \operatorname{Re}\{\mu\}>0$,

the average capacity is finally expressed as

$\bar{C}=\sum_{\substack{\mathcal{A} \subset \mathcal{S} \\ \mathcal{A} \neq \emptyset}} \frac{-(-1)^{|\mathcal{A}|}}{\ln 2}\left[a \mathrm{e}^{\beta} E_{1}(\beta)+\sum_{i \in \mathcal{A}} \frac{b_{i}}{\eta_{i}} E_{1}\left(\frac{\beta}{\eta_{i}}\right)\right]$

where $\beta=\sum_{i \in \mathcal{A}}\left(\frac{\lambda_{i, \mathrm{D}}}{P_{\mathrm{R}}}+\frac{\lambda_{\mathrm{S}, i}}{P_{\mathrm{S}}}\right)$ and $E_{i}(\cdot)$ is the exponential integral function.

When the link SNRs approach $\infty$, the exponential term in (18) becomes 1 and the average capacity can be evaluated as

$$
\bar{C}^{(\infty)}=\sum_{\substack{\mathcal{A} \subset \mathcal{S} \\ \mathcal{A} \neq \emptyset}} \frac{-(-1)^{|\mathcal{A}|}}{\ln 2} \int_{0}^{\infty} T(x) d x
$$

Using the identity $\int \frac{1}{b+a x} d x=\frac{1}{a} \ln |a x+b|$, the indefinite integral evaluates to:

$g(x)=\int T(x) d x=a \ln |1+x|+\sum_{i \in \mathcal{A}} \frac{b_{i}}{\eta_{i}} \ln \left|1+\eta_{i} x\right|+C$. 
Furthermore, by comparing $T(x)$ in (19) and (20) at $x \rightarrow$ $\infty$, it can be shown that $a+\sum_{i \in \mathcal{A}} \frac{b_{i}}{\eta_{i}}=0$. Using this relationship, $g(\infty)$ is calculated as

$$
\begin{aligned}
g(\infty) & =\lim _{x \rightarrow \infty} \sum_{i \in \mathcal{A}} \frac{b_{i}}{\eta_{i}}\left[\ln \left|1+\eta_{i} x\right|-\ln |1+x|\right] \\
& =\lim _{x \rightarrow \infty} \sum_{i \in \mathcal{A}} \frac{b_{i}}{\eta_{i}}\left[\ln \left|\frac{1+\eta_{i} x}{1+x}\right|\right]=\sum_{i \in \mathcal{A}} \frac{b_{i}}{\eta_{i}} \ln \eta_{i} .
\end{aligned}
$$

Finally, substituting (26) and $g(0)=0$ into (24) yields

$$
\bar{C}^{(\infty)}=\sum_{\substack{\mathcal{A} \subset \mathcal{S} \\ \mathcal{A} \neq \emptyset}} \frac{-(-1)^{|\mathcal{A}|}}{\ln 2}\left[\sum_{i \in \mathcal{A}} \frac{b_{i}}{\eta_{i}} \ln \left(\eta_{i}\right)\right] .
$$

The above analysis reveals that there is a hard limit on the capacity even when the transmit powers at the source and selected relay are the same and increase without bound. Again, this is a direct consequence of the nonzero residual self interference.

\section{Performance analysis under FAC and with power scaling}

The analysis in the previous section for the case when equal power is assigned at the source and selected relay, i.e., $P_{S}=P_{R}$, shows that the diversity order is zero and there is a hard limit on the capacity in the high SNR regime. This section analyzes the system performance in which power scaling is performed at the source in order to overcome zero diversity order and remove the hard capacity limit. The power scaling considered here is similar to what investigated in [19], but it is pointed out that only relay selection, not joint relay-antenna selection, is examined in [19]. In particular, the source power is scaled according to the instantaneous channel between the source and selected relay and the transmit power of the relay as follows:

$$
\hat{P}_{\mathrm{S}}=P_{\mathrm{R}}^{2}\left|h_{\mathrm{S}, i}^{(m)}\right|^{2} \text {. }
$$

With the above power scaling, the instantaneous SINR at relay $i$ becomes

$$
\hat{\gamma}_{\mathrm{S}, i}^{l \rightarrow m}=\frac{P_{\mathrm{R}}^{2}\left|h_{\mathrm{S}, i}^{(m)}\right|^{4}}{P_{\mathrm{R}}\left|I_{i, i}^{l \rightarrow m}\right|^{2}+1}=\frac{Z}{Y+1},
$$

where $Z=P_{\mathrm{R}}^{2}\left|h_{\mathrm{S}, i}^{(m)}\right|^{4}$ and $Y=P_{\mathrm{R}}\left|I_{i, i}^{l \rightarrow m}\right|^{2}$. The CDF of $Z$ and the PDF of $Y$ are given as follows:

$$
\begin{aligned}
& F_{Z}(z)=1-\exp \left(-\frac{\lambda_{\mathrm{S}, i}}{P_{\mathrm{R}}} \sqrt{z}\right) \\
& f_{Y}(y)=\frac{\lambda_{i, i}}{P_{\mathrm{R}}} \exp \left(-\frac{\lambda_{i, i}}{P_{\mathrm{R}}} y\right)
\end{aligned}
$$

\subsection{Outage probability}

In this case, the outage probability is derived as

$$
P_{\text {out }}(x)=\prod_{i=1}^{K} F_{l, m, i}(x),
$$

where

$$
\begin{aligned}
F_{l, m, i}(x) & =1-\left(1-F_{\hat{\gamma}_{\mathrm{S}, i}^{l \rightarrow m}}(x)\right)\left(1-F_{\gamma_{i, \mathrm{D}}^{l \rightarrow m}}(x)\right) \\
F_{\gamma_{i, \mathrm{D}}^{l \rightarrow m}}(x) & =1-\exp \left(-\frac{\lambda_{i, \mathrm{D}}}{P_{\mathrm{R}}} x\right) \\
F_{\hat{\gamma}_{\mathrm{S}, i}^{l \rightarrow m}}(x) & =\operatorname{Pr}\left(\frac{Z}{Y+1}<x\right)=\operatorname{Pr}(Z<(Y+1) x) \\
& =\int_{0}^{\infty} \operatorname{Pr}(Z<(Y+1) x) f_{Y}(y) d y \\
& =1-\frac{\lambda_{i, i}}{P_{\mathrm{R}}} \int_{0}^{\infty} \exp \left(-\frac{\lambda_{\mathrm{S}, i}}{P_{\mathrm{R}}} \sqrt{x} \sqrt{y+1}-\frac{\lambda_{i, i}}{P_{\mathrm{R}}} y\right) d y .
\end{aligned}
$$

The above integral can be evaluated in a closed form by making change of variable $u=\sqrt{y+1}$ and using the following identity [25]:

$$
\begin{aligned}
\int_{1}^{\infty} x \exp ( & \left.-m x-n x^{2}\right) d x \\
& =\frac{\exp (-(m+n))}{2 n} \\
& -\frac{\sqrt{\pi} m \exp \left(\frac{m^{2}}{4 n}\right) Q\left(\frac{\sqrt{2} m+2 \sqrt{2} n}{2 \sqrt{n}}\right)}{2 n^{\frac{3}{2}}} .
\end{aligned}
$$

The final expression for $F_{l, m, i}(x)$ is given as

$$
\begin{aligned}
F_{l, m, i}(x)= & 1-\exp \left(-\left(\frac{\lambda \mathrm{s}, i \sqrt{x}}{P_{\mathrm{R}}}+\frac{\lambda_{i, \mathrm{D}} x}{P_{\mathrm{R}}}\right)\right) \\
+ & \frac{\exp \left(\frac{\lambda_{i, i}}{P_{\mathrm{R}}}+\frac{\left(\lambda_{\mathrm{S}, i}\right)^{2} x}{4 P_{\mathrm{R}} \lambda_{i, i}}\right) \exp \left(-\frac{\lambda_{i, \mathrm{D}}}{P_{\mathrm{R}}} x\right) \frac{\lambda_{\mathrm{S}, i}}{P_{\mathrm{R}}} \sqrt{\pi x}}{\sqrt{\frac{\lambda_{i, i}}{P_{\mathrm{R}}}}} \\
& Q\left(\frac{\frac{\sqrt{2} \lambda_{\mathrm{S}, i} \sqrt{x}}{P_{\mathrm{R}}}+2 \sqrt{2} \frac{\lambda_{i, i}}{P_{\mathrm{R}}}}{2 \sqrt{\frac{\lambda_{i, i}}{P_{\mathrm{R}}}}}\right)
\end{aligned}
$$

By using the inequality $Q(x) \leq \frac{1}{2} \exp \left(-\frac{x^{2}}{2}\right)$, an upper bound on the outage probability is obtained as

$$
P_{\text {out }}(x) \leq P_{\text {out }}^{(\mathrm{UB})}(x)=\prod_{i=1}^{K} G_{l, m, i}(x)
$$

where $q_{i}$ and $G_{l, m, i}(x)$ are defined as 


$$
\begin{aligned}
q_{i}= & \frac{\lambda_{\mathrm{S}, i} \sqrt{\pi P_{\mathrm{R}}}}{2 P_{\mathrm{R}} \sqrt{\lambda_{i, i}}} \\
G_{l, m, i}(x)= & 1-\exp \left(-\left(\frac{\lambda_{\mathrm{S}, i}}{P_{\mathrm{R}}} \sqrt{x}+\frac{\lambda_{i, \mathrm{D}}}{P_{\mathrm{R}}} x\right)\right) \\
& +q_{i} \sqrt{x} \exp \left(-\left(\frac{\lambda_{\mathrm{R}, i} \sqrt{x}}{P_{\mathrm{R}}}+\frac{\lambda_{i, \mathrm{D}} x}{P_{\mathrm{R}}}\right)\right) .
\end{aligned}
$$

Furthermore, in the high SNR regime, $q_{i} \rightarrow 0$ and the outage probability can be upper bounded as

$P_{\text {out }}(x) \leq \prod_{i=1}^{K}\left(1-\exp \left(-\left(\frac{\lambda_{\mathrm{R}, i}}{P_{\mathrm{R}}} \sqrt{x}+\frac{\lambda_{i, \mathrm{D}}}{P_{\mathrm{R}}} x\right)\right)\right)$.

Using the definition of finite-SNR diversity order in (14), the diversity order can be obtained as

$d=\sum_{i=1}^{K} \frac{\left(\lambda_{\mathrm{S}, i} \sqrt{x}+\lambda_{i, \mathrm{D}} x\right) \exp \left(-\left(\frac{\lambda_{\mathrm{S}, i} \sqrt{x}}{P_{\mathrm{R}}}+\frac{\lambda_{i, \mathrm{D} x}}{P_{\mathrm{R}}}\right)\right)}{P_{\mathrm{R}}\left(1-\exp \left(-\left(\frac{\lambda_{\mathrm{S}, i} \sqrt{x}}{P_{\mathrm{R}}}+\frac{\lambda_{i, D} x}{P_{\mathrm{R}}}\right)\right)\right)}$.

In the high SNR regime, $1-\exp \left(-\left(\frac{\lambda_{S, i} \sqrt{x}}{P_{\mathrm{R}}}+\frac{\lambda_{i, \mathrm{D}} x}{P_{\mathrm{R}}}\right)\right) \approx$ $\left(\frac{\lambda_{\mathrm{S}, i} \sqrt{x}}{P_{\mathrm{R}}}+\frac{\lambda_{i, \mathrm{D} x}}{P_{\mathrm{R}}}\right)$. Therefore, the full diversity order $d=K$ can be achieved, regardless of the RSI level $\eta_{i}$.

The above analysis is with respect to the relay power, i.e., the SNR $\bar{\gamma}=\frac{P_{\mathrm{R}}}{\sigma_{w}^{2}}$. Note that, depending on the instantaneous channel gain $\left|h_{\mathrm{S}, i}^{(m)}\right|^{2}$, the instantaneous source power in (29) might be larger or smaller than $P_{\mathrm{R}}$. When the total power is constrained to be $P$, then

$$
\begin{aligned}
\frac{P_{\mathrm{R}}^{2}}{\lambda_{\mathrm{S}, i}}+P_{\mathrm{R}} & =P \\
P_{\mathrm{R}} & =\frac{-\lambda_{\mathrm{S}, i}+\sqrt{\lambda_{\mathrm{S}, i}^{2}+4 P \lambda_{\mathrm{S}, i}}}{2}
\end{aligned}
$$

Therefore, the diversity order with respect to the SNR $\bar{\gamma}=$ $\frac{P}{\sigma_{w}^{2}}$ is obtained as

$$
\frac{\partial \ln P_{\text {out }}}{\partial \ln P}=\frac{\partial \ln P_{\text {out }}}{\partial \ln P_{\mathrm{R}}} \times \frac{\partial \ln P_{\mathrm{R}}}{\partial P_{\mathrm{R}}} \times \frac{\partial P_{\mathrm{R}}}{\partial P} \times \frac{\partial P}{\partial \ln P}=\frac{K}{2} .
$$

Thus, in this case the diversity order is twice smaller. Therefore, there is a tradeoff between the diversity order and how the source power is scaled with respect to the total power.

\subsection{Average capacity}

The upper bound of outage probability in (39) can be written as

$$
\begin{aligned}
P_{\text {out }}^{(\mathrm{UB})}(x)= & \sum_{\substack{\mathcal{A} \cup \mathcal{B} \subset S \\
\mathcal{A} \cup \mathcal{B} \neq \emptyset \\
\mathcal{A} \cap \mathcal{B}=\emptyset}}\left[\prod_{i \in \mathcal{A}}\left(-\exp \left(-\left(\frac{\lambda_{\mathrm{S}, i}}{P_{\mathrm{R}}} \sqrt{x}+\frac{\lambda_{i, \mathrm{D}}}{P_{\mathrm{R}}} x\right)\right)\right)\right. \\
& \left.\times \prod_{j \in \mathcal{B}}\left(q_{j} \sqrt{x} \exp \left(-\left(\frac{\lambda_{\mathrm{S}, i}}{P_{\mathrm{R}}} \sqrt{x}+\frac{\lambda_{i, \mathrm{D}}}{P_{\mathrm{R}}} x\right)\right)\right)\right] .
\end{aligned}
$$

The corresponding lower bound on the average capacity can then be computed as follows:

$$
\begin{aligned}
& \bar{C}_{(\mathrm{LB})}=\frac{1}{\ln 2} \int_{0}^{\infty} \frac{1-P_{\mathrm{out}}^{(\mathrm{UB})}(x)}{1+x} d x \\
& =\sum_{\substack{\mathcal{A} \cup \mathcal{B} \subset S \\
\mathcal{A} \cup \mathcal{B} \neq \emptyset \\
\mathcal{A} \cap \mathcal{B}=\emptyset}} \frac{-(-1)^{|\mathcal{A}|} \prod_{j \in \mathcal{B}} q_{j}}{\ln 2} \int_{0}^{\infty} \frac{x^{\frac{|\mathcal{B}|}{2}}}{1+x} \\
& \exp \left(-\sum_{i \in \mathcal{S}}\left(\frac{\lambda_{i, \mathrm{D}}}{P_{\mathrm{R}}} x+\frac{\lambda_{\mathrm{S}, i}}{P_{\mathrm{R}}} \sqrt{x}\right)\right) d x
\end{aligned}
$$

where the summation is employed over all possible sets for $\mathcal{A}$ and $\mathcal{B}$ such that $\mathcal{A} \cup \mathcal{B} \in \mathcal{S}, \mathcal{A} \cup \mathcal{B} \neq \emptyset$ and $\mathcal{A} \cap \mathcal{B}=\emptyset$.

In the high SNR regime, the lower bound of the average capacity can be written as

$$
\bar{C}_{(\mathrm{LB})}^{(\infty)}=\sum_{\substack{\mathcal{A} \cup \mathcal{B} \subset S \\ \mathcal{A} \cup \mathcal{B} \neq \emptyset \\ \mathcal{A} \cap \mathcal{B}=\emptyset}} \frac{-(-1)^{|\mathcal{A}|} \prod_{j \in \mathcal{B}} q_{j}}{\ln 2} \int_{0}^{\infty} \frac{x^{\frac{|\mathcal{B}|}{2}}}{1+x} d x \rightarrow \infty
$$

where $|\mathcal{A}|$ and $|\mathcal{B}|$ are the cardinalities of sets $\mathcal{A}$ and $\mathcal{B}$. As can be seen, the lower bound increases without bound. Thus, there is no capacity limit when power scaling is performed.

\section{Performance analysis under AAC: with and without power scaling}

6.1 Without power scaling

Focusing on the case $Q=2$, define $\gamma_{i}$ as follows:

$\gamma_{i}=\max \left\{\min \left\{\gamma_{\mathrm{S}, i}^{l \rightarrow m}, \gamma_{i, \mathrm{D}}^{l \rightarrow m}\right\}, \min \left\{\gamma_{\mathrm{S}, i}^{m \rightarrow l}, \gamma_{i, \mathrm{D}}^{m \rightarrow l}\right\}\right\}$

For notational convenience, let $\tilde{\lambda}_{\mathrm{S}, i}=\lambda_{\mathrm{S}, i} / P_{\mathrm{S}}, \tilde{\lambda}_{i, \mathrm{D}}=$ $\lambda_{i, \mathrm{D}} / P_{\mathrm{R}}$, and $\tilde{\lambda}_{i, i}=\lambda_{i, i} / P_{\mathrm{R}}$. In essence, $\tilde{\lambda}_{\mathrm{S}, i}, \tilde{\lambda}_{i, \mathrm{D}}$, and $\tilde{\lambda}_{i, i}$ are the exponential parameters of scaled random variables $P_{\mathrm{S}}\left|h_{\mathrm{S}, i}^{(m)}\right|^{2}, P_{\mathrm{R}}\left|h_{i, \mathrm{D}}^{(l)}\right|^{2}$, and $P_{\mathrm{R}}\left|I_{i, i}^{l \rightarrow m}\right|^{2}$, respectively. According to the including-excluding principle [18], the CDF of $\gamma_{i}$ can be obtained as in (52). 


$$
\begin{aligned}
& F_{\gamma_{i}}(x)=\operatorname{Pr}\left(\min \left\{\frac{P_{\mathrm{S}}\left|h_{\mathrm{S}, i}^{(m)}\right|^{2}}{P_{\mathrm{R}}\left|I_{i, i}^{l \rightarrow m}\right|^{2}+1}, P_{R}\left|h_{i, \mathrm{D}}^{(l)}\right|^{2}\right\}\right. \\
& \left.<x, \min \left\{\frac{P_{\mathrm{S}}\left|h_{\mathrm{S}, i}^{(m)}\right|^{2}}{P_{R}\left|I_{i, i}^{m \rightarrow l}\right|^{2}+1}, P_{\mathrm{R}}\left|h_{i, \mathrm{D}}^{(l)}\right|^{2}\right\}<x\right) \\
& =\operatorname{Pr}\left\{\min \left\{\frac{P_{\mathrm{S}}\left|h_{\mathrm{S}, i}^{(m)}\right|^{2}}{P_{\mathrm{R}}\left|I_{i, i}^{l l \rightarrow m}\right|^{2}+1}, P_{\mathrm{R}}\left|h_{i, \mathrm{D}}^{(l)}\right|^{2}\right\}\right. \\
& \left.>x, \min \left\{\frac{P_{\mathrm{S}}\left|h_{\mathrm{S}, i}^{(m)}\right|^{2}}{P_{\mathrm{R}}\left|I_{i, i}^{m \rightarrow l}\right|^{2}+1}, P_{\mathrm{R}}\left|h_{i, \mathrm{D}}^{(l)}\right|^{2}\right\}>x\right) \\
& +1-\operatorname{Pr}\left(\min \left\{\frac{P_{\mathrm{S}}\left|h_{\mathrm{S}, i}^{(m)}\right|^{2}}{P_{\mathrm{R}}\left|I_{i, i}^{l-m}\right|^{2}+1}, P_{\mathrm{R}}\left|h_{i, \mathrm{D}}^{(l)}\right|^{2}\right\}>x\right) \\
& -\operatorname{Pr}\left(\min \left\{\frac{P_{\mathrm{S}}\left|h_{\mathrm{S}, i}^{(m)}\right|^{2}}{P_{\mathrm{R}}\left|I_{i, i}^{m \rightarrow l}\right|^{2}+1}, P_{\mathrm{R}}\left|h_{i, \mathrm{D}}^{(l)}\right|^{2}\right\}>x\right) \\
& =1-\frac{2 \exp \left(-\left(\tilde{\lambda}_{S, i}+\tilde{\lambda}_{i, \mathrm{D}}\right) x\right)}{1+x \eta_{i}} \\
& +\frac{\exp \left(-2\left(\tilde{\lambda}_{\mathrm{S}, i}+\tilde{\lambda}_{i, \mathrm{D}}\right) x\right)}{1+2 \eta_{i} x} .
\end{aligned}
$$

Next, define $\gamma_{\max }=\max \left\{\gamma_{1}, \ldots, \gamma_{K}\right\}$. Then, the CDF of $\gamma_{\max }$ is $F_{\gamma_{\max }}(x)=\prod_{i=1}^{K} F_{\gamma_{i}}(x)$, which is also the outage performance of the system. Based on the definition of finite-SNR diversity order, it is obtained as in (53).

$$
\begin{aligned}
d & =\sum_{i=1}^{K} \frac{2 x}{P_{\mathrm{R}}}\left(\lambda_{S, i}+\lambda_{i, \mathrm{D}}\right) \\
& \times \frac{\frac{1}{1+\eta_{i x} x}\left(\exp \left(-\left(\frac{\lambda_{S, i}}{P_{S}}+\frac{\lambda_{i, \mathrm{D}}}{P_{\mathrm{R}}}\right) x\right)\right)-\frac{1}{1+2 \eta_{i x} x}\left(\exp \left(-2\left(\frac{\lambda_{S, i}}{P_{S}}+\frac{\lambda_{i, \mathrm{D}}}{P_{\mathrm{R}}}\right) x\right)\right)}{1-\frac{2}{1+\eta_{i} x}\left(\exp \left(-\left(\frac{\lambda_{S, i}}{P_{\mathrm{S}}}+\frac{\lambda_{i, \mathrm{D}}}{P_{\mathrm{R}}}\right) x\right)\right)+\frac{1}{1+2 \eta_{i x} x}\left(\exp \left(-2\left(\frac{\lambda_{S, i}}{P_{S}}+\frac{\lambda_{i, \mathrm{D}}}{P_{\mathrm{R}}}\right) x\right)\right)}
\end{aligned}
$$

By employing Taylor series expansion, the diversity order in this case can be approximated as

$$
d \approx \sum_{i=1}^{K} 2 \frac{1+\zeta_{i}}{1+2 \zeta_{i}+2 \zeta_{i}^{2}}
$$

where $\zeta_{i}=\frac{P_{\mathrm{R}} \eta_{i}}{\lambda_{\mathrm{S}, i}+\lambda_{i, \mathrm{D}}}$. It can be seen that as $\eta_{i} \rightarrow 0, \zeta_{i} \rightarrow 0$ and $d \rightarrow 2 K$. Thus, by the fact that having adaptive configuration between the transmit and receive antennas, the number of effective channels between the source and $K$ relays or between $K$ relays and the destination becomes
$2 K$, which explains the diversity order $d \rightarrow 2 K$ when the RSI approaches zero.

Next, rewrite $F_{\gamma_{\max }}(x)$ as

$$
\begin{array}{r}
F_{\gamma_{\max }}(x)=\sum_{\substack{\mathcal{A} \cup \mathcal{B} \subset S \\
\mathcal{A} \cap \mathcal{B}=\emptyset}}\left[\prod_{i \in \mathcal{A}}\left(-2 \frac{\exp \left(-\left(\tilde{\lambda}_{\mathrm{S}, i}+\tilde{\lambda}_{i, \mathrm{D}}\right) x\right)}{1+x \eta_{i}}\right)\right. \\
\left.\prod_{j \in \mathcal{B}}\left(\frac{\exp \left(-2\left(\tilde{\lambda}_{\mathrm{S}, i}+\tilde{\lambda}_{i, \mathrm{D}}\right) x\right)}{1+2 x \eta_{i}}\right)\right]
\end{array}
$$

where $\mathcal{A}, \mathcal{B}$, and $\mathcal{S}$ were defined as before. Therefore, the average capacity is

$$
\begin{aligned}
\bar{C}= & \sum_{\substack{\mathcal{A} \cap \mathcal{B}=\emptyset \\
\mathcal{A} \cup \mathcal{B} \subset \boldsymbol{\mathcal { B }} \\
\mathcal{A} \cup \mathcal{B} \neq \emptyset}} \frac{-(-2)^{|\mathcal{A}|}}{\ln 2} \int_{0}^{\infty} H(x) \\
& \times \exp \left(-\left(\sum_{i \in \mathcal{A}}\left(\tilde{\lambda}_{\mathrm{S}, i}+\tilde{\lambda}_{i, \mathrm{D}}\right)+2 \sum_{j \in \mathcal{B}}\left(\tilde{\lambda}_{\mathrm{S}, j}+\tilde{\lambda}_{j, \mathrm{D}}\right)\right) x\right) d x \\
= & \sum_{\substack{\mathcal{A} \cap \mathcal{B}=\emptyset \\
\mathcal{A} \cup \mathcal{B} \subset S}} \frac{-(-2)^{|\mathcal{A}|}}{\ln 2}\left(a \exp (\mu)+\sum_{i \in \mathcal{A}} \frac{b_{i}}{\eta_{i}} E_{1}\left(\frac{\mu}{\eta_{i}}\right)\right. \\
& \left.+\sum_{j \in \mathcal{B}} \frac{c_{j}}{2 \eta_{j}} E_{1}\left(\frac{\mu}{2 \eta_{j}}\right)\right)
\end{aligned}
$$

where $H(x), a, b_{i}, c_{j}$, and $\mu$ are

$$
\begin{aligned}
H(x) & =\frac{1}{(1+x) \prod_{i \in \mathcal{A}}\left(1+\eta_{i} x\right) \prod_{j \in \mathcal{B}}\left(1+2 \eta_{j} x\right)} \\
& =\frac{a}{1+x}+\sum_{i \in \mathcal{A}} \frac{b_{i}}{1+\eta_{i} x}+\sum_{j \in \mathcal{B}} \frac{c_{j}}{1+2 \eta_{j} x} \\
a & =\frac{1}{\prod_{i \in \mathcal{A}}\left(1-\eta_{i}\right) \prod_{j \in \mathcal{B}}\left(1-2 \eta_{j}\right)} \\
b_{i} & =\frac{1}{\left(1-\frac{1}{\eta_{i}}\right) \prod_{\substack{i^{\prime} \in \mathcal{A} \\
i^{\prime} \neq i}}\left(1-\frac{\eta_{i^{\prime}}}{\eta_{i}}\right) \prod_{j \in \mathcal{B}}\left(1-\frac{2 \eta_{j}}{\eta_{i}}\right)} \\
c_{j} & =\frac{1}{\left(1-\frac{1}{2 \eta_{j}}\right) \prod_{\substack{i \in \mathcal{A} \\
\left(1-\frac{\eta_{i}}{2 \eta_{j}}\right)}} \prod_{\substack{j^{\prime} \in \mathcal{B} \\
j^{\prime} \neq j}}\left(1-\frac{\eta_{j^{\prime}}}{\eta_{j}}\right)} \\
\mu & =\sum_{i \in \mathcal{A}}\left(\tilde{\lambda}_{\mathrm{S}, i}+\tilde{\lambda}_{i, \mathrm{D}}\right)+2 \sum_{j \in \mathcal{B}}\left(\tilde{\lambda}_{\mathrm{S}, j}+\tilde{\lambda}_{j, \mathrm{D}}\right)
\end{aligned}
$$




\subsection{With power scaling}

Similar to the case of fixed antenna configuration, when power scaling is employed in adaptive antenna configuration, define

$\hat{\gamma}_{i}=\max \left\{\min \left\{\hat{\gamma}_{\mathrm{S}, i}^{l \rightarrow m}, \gamma_{i, \mathrm{D}}^{l \rightarrow m}\right\}, \min \left\{\hat{\gamma}_{\mathrm{S}, i}^{m \rightarrow l}, \gamma_{i, \mathrm{D}}^{m \rightarrow l}\right\}\right\}$,

where $\hat{\gamma}_{\mathrm{S}, i}^{l \rightarrow m}$ is defined as in (30). Then the CDF of $\hat{\gamma}_{i}$ can be derived similarly as done in (52) and the result is given in (63), where $\vartheta=\left|I_{i, i}^{l \rightarrow m}\right|^{2}$ implies a specific value of random variable $\left|I_{i, i}^{l \rightarrow m}\right|^{2}$.

$$
\begin{aligned}
& F_{\hat{\gamma}_{i}}(x)=\operatorname{Pr}\left(\min \left\{\frac{\hat{P}_{\mathrm{S}}}{P_{\mathrm{R}}\left|I_{i, i}^{l \rightarrow m}\right|^{2}+1}, P_{\mathrm{R}}\left|h_{i, \mathrm{D}}^{(l)}\right|^{2}\right\}\right. \\
& \left.<x, \min \left\{\frac{\hat{P}_{\mathrm{S}}}{P_{\mathrm{R}}\left|I_{i, i}^{m \rightarrow l}\right|^{2}+1}, P_{\mathrm{R}}\left|h_{i, \mathrm{D}}^{(l)}\right|^{2}\right\}<x\right) \\
& =\operatorname{Pr}\left(\min \left\{\frac{\hat{P}_{\mathrm{S}}}{P_{\mathrm{R}}\left|I_{i, i}^{l \rightarrow m}\right|^{2}+1}, P_{\mathrm{R}}\left|h_{i, \mathrm{D}}^{(l)}\right|^{2}\right\}\right. \\
& \left.>x, \min \left\{\frac{\hat{P}_{\mathrm{S}}}{P_{\mathrm{R}}\left|I_{i, i}^{m \rightarrow l}\right|^{2}+1}, P_{\mathrm{R}}\left|h_{i, \mathrm{D}}^{(l)}\right|^{2}\right\}>x\right) \\
& +1-\operatorname{Pr}\left(\min \left\{\frac{\hat{P}_{\mathrm{S}}}{P_{\mathrm{R}}\left|I_{i, i}^{l \rightarrow m}\right|^{2}+1}, P_{\mathrm{R}}\left|h_{i, \mathrm{D}}^{(l)}\right|^{2}\right\}>x\right) \\
& -\operatorname{Pr}\left(\min \left\{\frac{\hat{P}_{\mathrm{S}}}{P_{\mathrm{R}}\left|I_{i, i}^{m \rightarrow l}\right|^{2}+1}, P_{\mathrm{R}}\left|h_{i, \mathrm{D}}^{(l)}\right|^{2}\right\}>x\right) \\
& =1-2 \exp \left(-\tilde{\lambda}_{i, \mathrm{D}} x\right) \int_{0}^{\infty} \exp \left(-\tilde{\lambda}_{i, i} \vartheta-\sqrt{(\vartheta+1) x} \tilde{\lambda}_{\mathrm{s}, i}\right) d \vartheta \\
& +\exp \left(-2 \tilde{\lambda}_{i, \mathrm{D} x)} \int_{0}^{\infty} \exp \left(-\tilde{\lambda}_{i, i} \vartheta-2 \sqrt{(\vartheta+1) x} \tilde{\lambda}_{s, i}\right) d \vartheta\right. \\
& =1-2 \exp \left(-\left(\tilde{\lambda}_{i, \mathrm{D}} x+\tilde{\lambda}_{\mathrm{S}, i} \sqrt{x}\right)\right) \\
& +4 \frac{\sqrt{\pi x} \tilde{\lambda}_{\mathrm{S}, i} \exp \left(\frac{\tilde{\lambda}_{\mathrm{S}, i}^{2} x}{4 \tilde{\lambda}_{i, i}}+\tilde{\lambda}_{i, i}-\tilde{\lambda}_{i, \mathrm{D}} x\right) Q\left(\frac{\sqrt{2} \tilde{\lambda}_{\mathrm{S}, i} \sqrt{x}+\sqrt{2} \tilde{\lambda}_{i, i}}{2 \sqrt{\tilde{\lambda}_{i, i}}}\right)}{\sqrt{\tilde{\lambda}_{i, i}}} \\
& +\exp \left(-2\left(\tilde{\lambda}_{i, \mathrm{D} x}+\tilde{\lambda}_{S, i} \sqrt{x}\right)\right) \\
& -4 \frac{\sqrt{\pi x} \tilde{\lambda}_{\mathrm{S}, i} \exp \left(\frac{\tilde{\lambda}_{\mathrm{S}, i}^{2} x}{\tilde{\lambda}_{i, i}}+\tilde{\lambda}_{i, i}-2 \tilde{\lambda}_{i, \mathrm{D}} x\right) Q\left(\frac{\sqrt{2} \tilde{\lambda}_{\mathrm{S}, i} \sqrt{x}+\sqrt{2} \tilde{\lambda}_{i, i}}{\sqrt{\tilde{\lambda}_{i, i}}}\right)}{\sqrt{\tilde{\lambda}_{i, i}}}
\end{aligned}
$$

Finally, define $\hat{\gamma}_{\max }=\max \left\{\hat{\gamma}_{1}, \ldots, \hat{\gamma}_{K}\right\}$. Then, the outage probability is simply calculated as

$$
P_{\text {out }}(x)=F_{\hat{\gamma}_{\text {max }}}(x)=\prod_{i=1}^{K} F_{\hat{\gamma}_{i}}(x) .
$$

In the high transmit power scenario, the outage probability can be written as

$$
\begin{aligned}
P_{\text {out }}(x) \approx & \prod_{i=1}^{K}\left(1-2 \exp \left(-\left(\frac{\lambda_{\mathrm{S}, i} \sqrt{x}}{P_{\mathrm{S}}}+\frac{\lambda_{i, \mathrm{D} x}}{P_{\mathrm{R}}}\right)\right)\right. \\
& \left.+\exp \left(-2\left(\frac{\lambda_{\mathrm{S}, i} \sqrt{x}}{P_{\mathrm{S}}}+\frac{\lambda_{i, \mathrm{D} x}}{P_{\mathrm{R}}}\right)\right)\right)
\end{aligned}
$$

Based on the definition of finite-SNR diversity order, it is obtained as in (66).

$$
\begin{aligned}
d= & \sum_{i=1}^{K} \frac{2}{P_{\mathrm{R}}}\left(\lambda_{\mathrm{S}, i} \sqrt{x}+\lambda_{i, \mathrm{D} x}\right) \\
& \times \frac{\exp \left(-\left(\frac{\lambda_{\mathrm{S}, i} \sqrt{x}}{P_{\mathrm{S}}}+\frac{\lambda_{i, \mathrm{D}} x}{P_{\mathrm{R}}}\right)\right)-\exp \left(-2\left(\frac{\lambda_{\mathrm{S}, i} \sqrt{x}}{P_{\mathrm{S}}}+\frac{\lambda_{i, \mathrm{D}} x}{P_{\mathrm{R}}}\right)\right)}{1-\exp \left(-\left(\frac{\lambda_{\mathrm{S}, i} \sqrt{x}}{P_{\mathrm{S}}}+\frac{\lambda_{i, \mathrm{D}} x}{P_{\mathrm{R}}}\right)\right)+\exp \left(-2\left(\frac{\lambda_{\mathrm{S}, i} \sqrt{x}}{P_{\mathrm{S}}}+\frac{\lambda_{i, \mathrm{D} x} x}{P_{\mathrm{R}}}\right)\right)}
\end{aligned}
$$

Again, in the high transmit power scenario, employing the Taylor series expansion can easily show that $d \rightarrow 2 K$.

\section{Results and discussion}

In this section, numerical results are given to corroborate the theoretical analysis carried out in previous sections. Without loss of generality, suppose that all channels have unit power gains, i.e., $\lambda_{\mathrm{S}, i}=\lambda_{i, \mathrm{D}}=1, i=1, \ldots, K$. When no power scaling is performed, the transmit powers of the source and selected relay are set to be equal, i.e., $P_{\mathrm{S}}=P_{\mathrm{R}}$. Throughout this section, AWGN noise power is set to unity.

Figure 2 plots the finite-SNR diversity orders of the proposed joint relay-antenna selection schemes under both cases of FAC and AAC and with different self-interference levels and $K=3$. Observe that when there is nonzero selfinterference, the finite-SNR diversity order under either AAC or FAC approaches zero in the high transmit power region. In the low-to-medium transmit power region the diversity order under AAC is always greater than that under FAC. As expected, in the absence of the selfinterference at relay nodes, the diversity order under AAC approaches $2 K$, whereas the diversity order under FAC is $K$.

Figure 3 shows the outage performance versus the transmit power for the considered FD relay systems with $K=$ $2,3,5$ and the self-interference level $\eta=0.01$. The performance is included under both cases of FAC and AAC. As can be seen, the outage probability obtained by simulation matches very well with the expressions in (13) and (52). It can also be seen from the figure that there is a performance floor which agrees with the theoretical analysis. Under the same self-interference condition, the outage performance under AAC outperforms that under FAC. Also, as the number of relays increases, the outage performance gets better. 


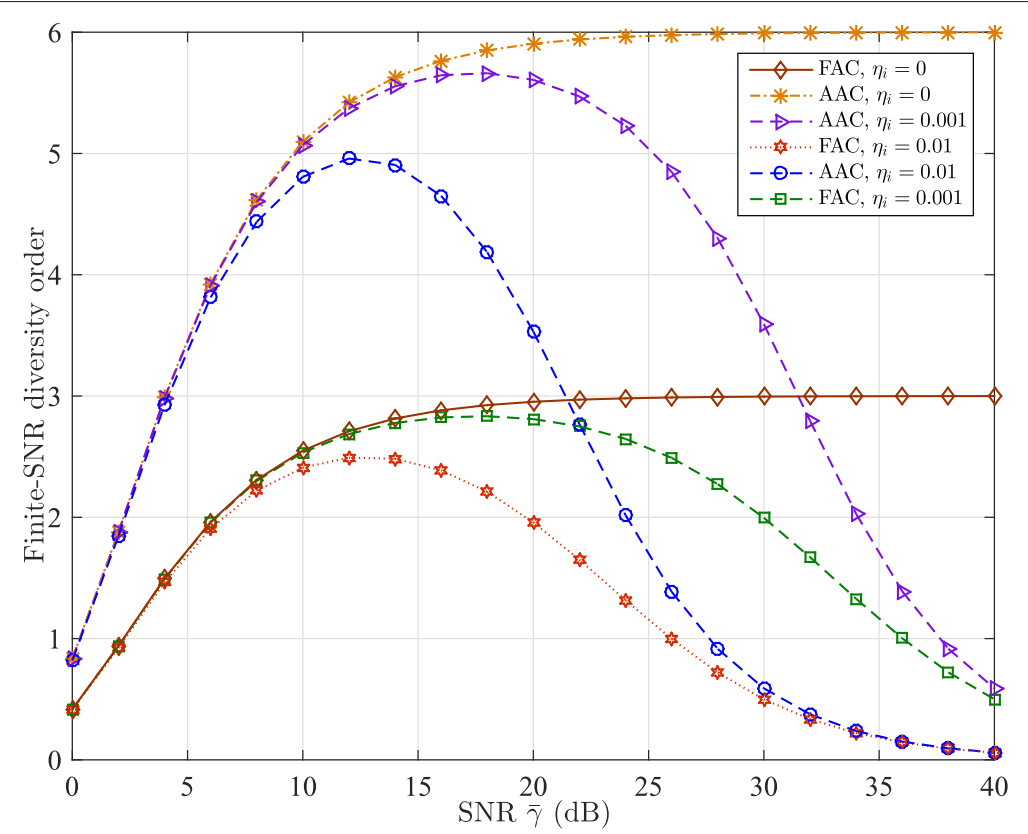

Fig. 2 Finite-SNR diversity orders of the proposed joint relay-antenna selection schemes versus the transmit power with different self-interference levels for $K=3$

Figure 4 shows the outage probability under AAC with the proposed power scaling for different values of $\lambda_{i, i}$ and when $K=3$. Recall that with the proposed power scaling, the transmit power of the source varies with the instantaneous CSI. For comparison, performance under AAC scheme with equal power allocation between the source and selected relay is also plotted. For all values of the self-interference, the system with power scaling achieves higher diversity order. Specifically, compared to the case of $\mathrm{FAC}$, the case of $\mathrm{AAC}$ with equal power achieves twice the diversity order at low-to-medium SNRs and a much lower outage floor at high SNRs. This figure also shows that the outage probability under AAC and with power scaling changes very little with the increase of $\lambda_{i, i}$. This

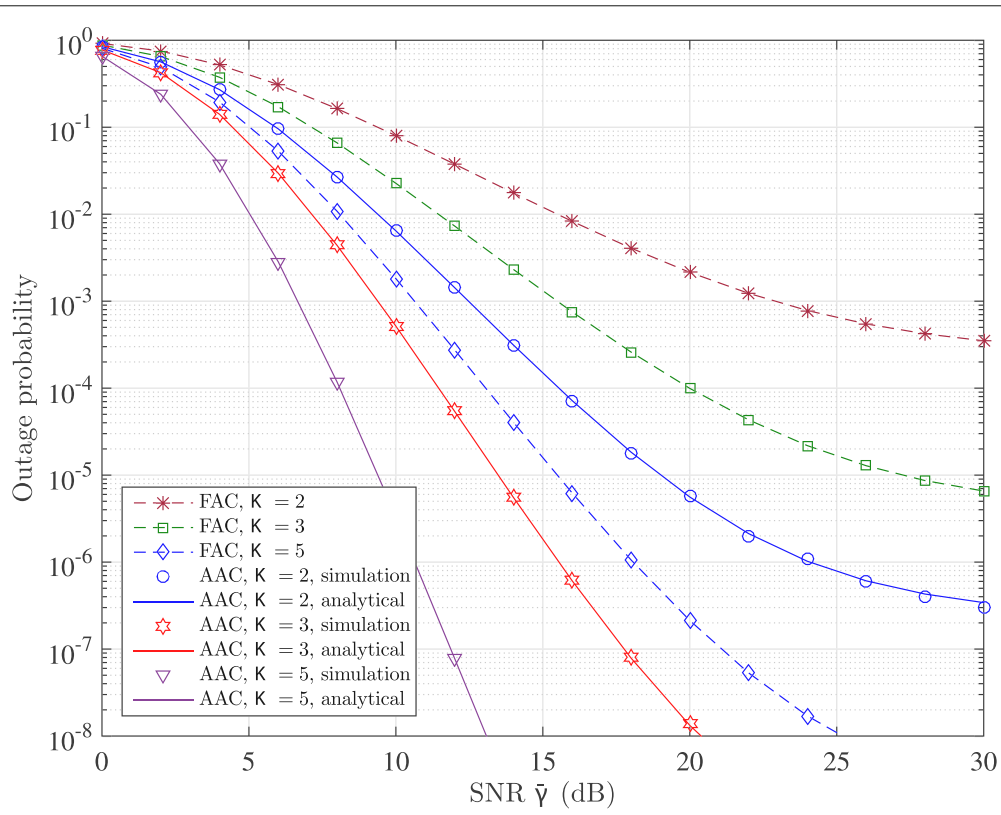

Fig. 3 Outage performance versus transmit power for the FD relay system with different values of $K$ and the self-interference level $\eta=0.01$ 


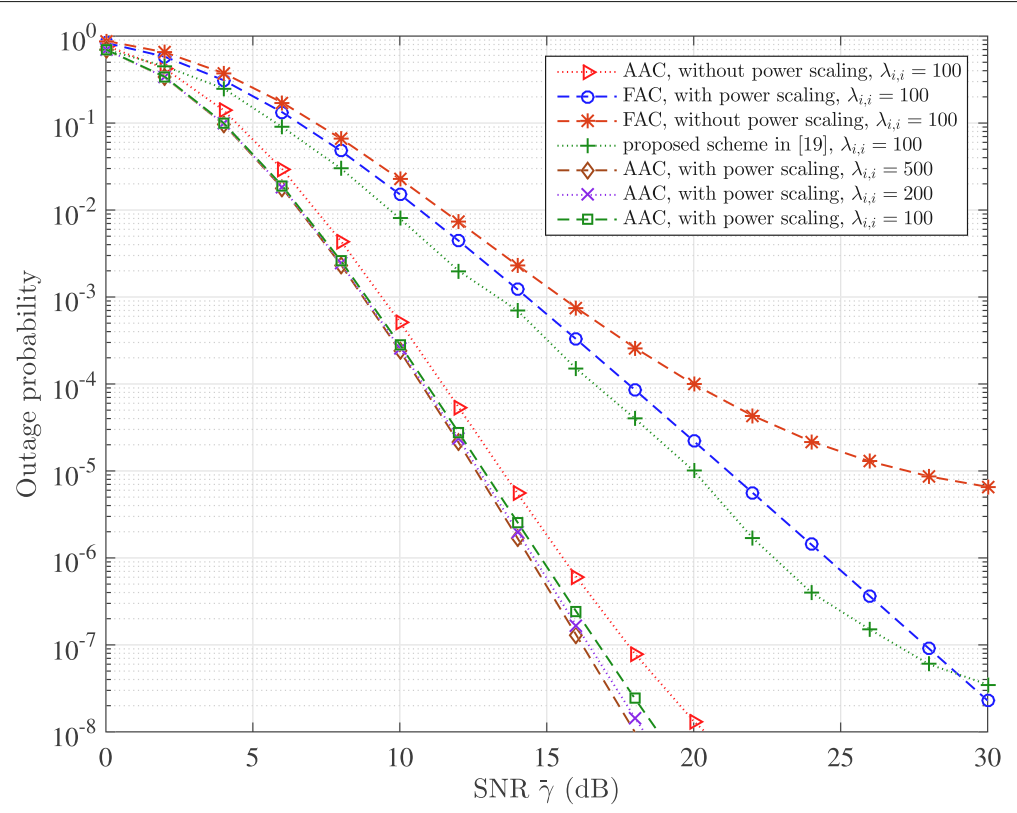

Fig. 4 Outage probability of the proposed joint relay-antenna selection versus transmit power with different values of $\lambda_{i, i}$ and $K=3$

means that the proposed scheme is robust to the variation of self interference. In fact, the diversity order of the proposed scheme under AAC and with power scaling is equal to $2 K$ even when $\lambda_{i, i}$ changes. Therefore, the diversity order is not influenced by the self-interference under $\mathrm{AAC}$ and with the proposed power scaling. Furthermore, performance of the conventional FD system considering the availability of the direct link, proposed in [19], is also included in this figure. As can be seen, our considered FD system under AAC (with or without power scaling), even without the availability of the direct link, outperforms the system in [19].

The ergodic capacity is shown in Fig. 5 under AAC (with and without power scaling) with $K=2$ and $\lambda_{i, i}=$

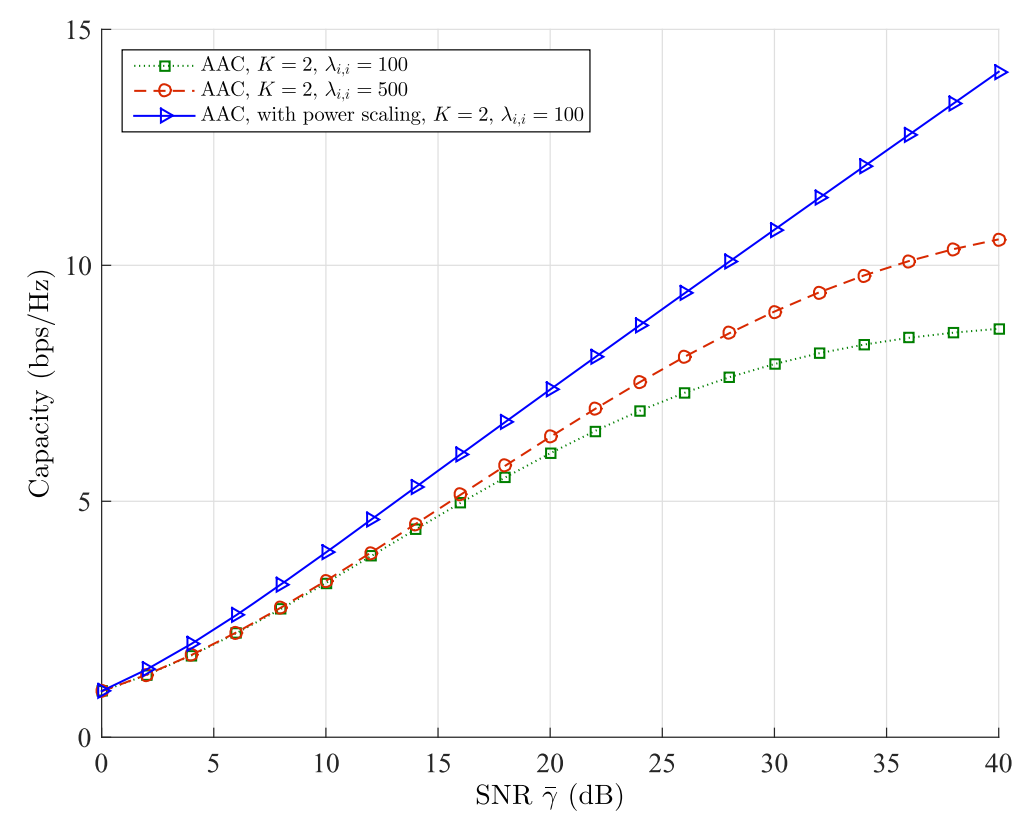

Fig. 5 Ergodic capacity under AAC with $K=2$ and different values of $\lambda_{i, i}$ 


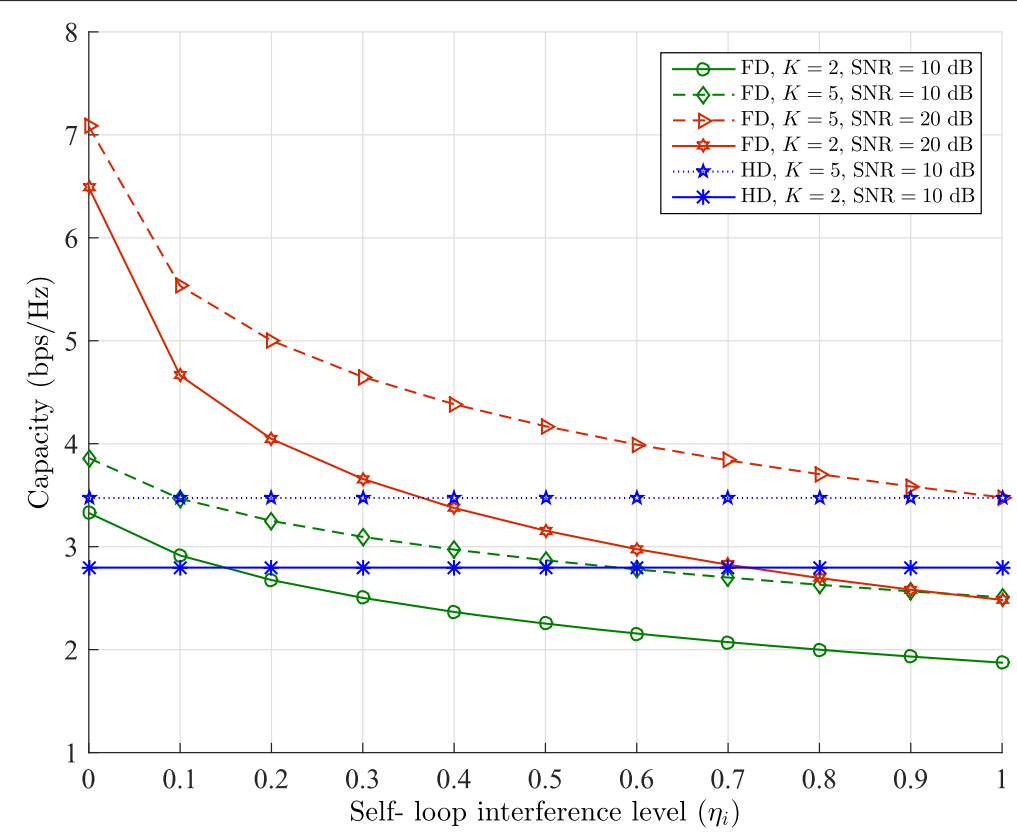

Fig. 6 Capacity performance of the FD and HD relay systems versus $\eta_{i}$

100,500. It can be seen that when $\lambda_{i, i}$ increases, the ergodic capacity under AAC improves. There is a capacity ceiling for the case of AAC without power scaling, whereas under AAC and with power scaling, capacity ceiling does not exist, which agrees with the analytical results obtained in previous sections.

Figure 6 compares the capacity performance of FD and HD relay systems obtained by simulation versus $\eta_{i}$. The transmit power of the source and relay is considered equal in both FD and HD systems. The performance of the FD relay system with AAC is simulated for two different SNRs. The simulation results indicate that when the self-interference is small, the capacity performance of the FD relay system outperforms that of the HD relay system. Also, as SNR becomes larger, the capacity performance of both FD and HD systems can be improved.

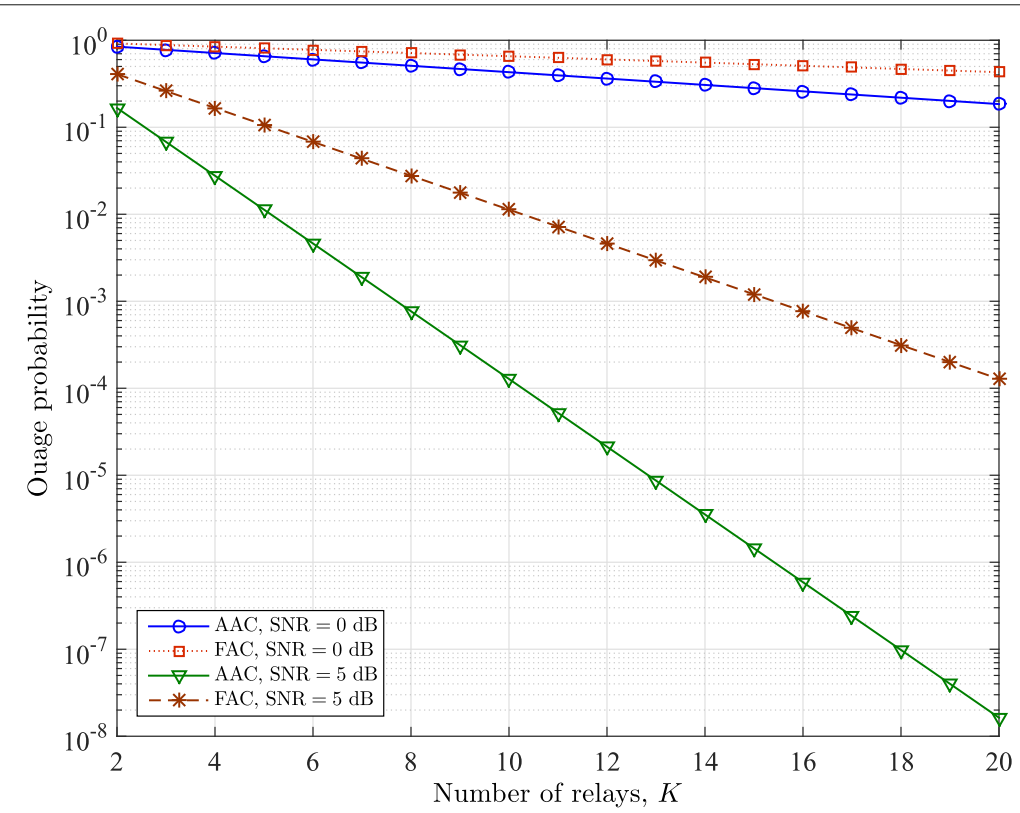

Fig. 7 Outage performance comparison for AAC and FAC versus the number of relays $K$ for different SNR values $(0,5 \mathrm{~dB})$ at $\eta=0.01$ 


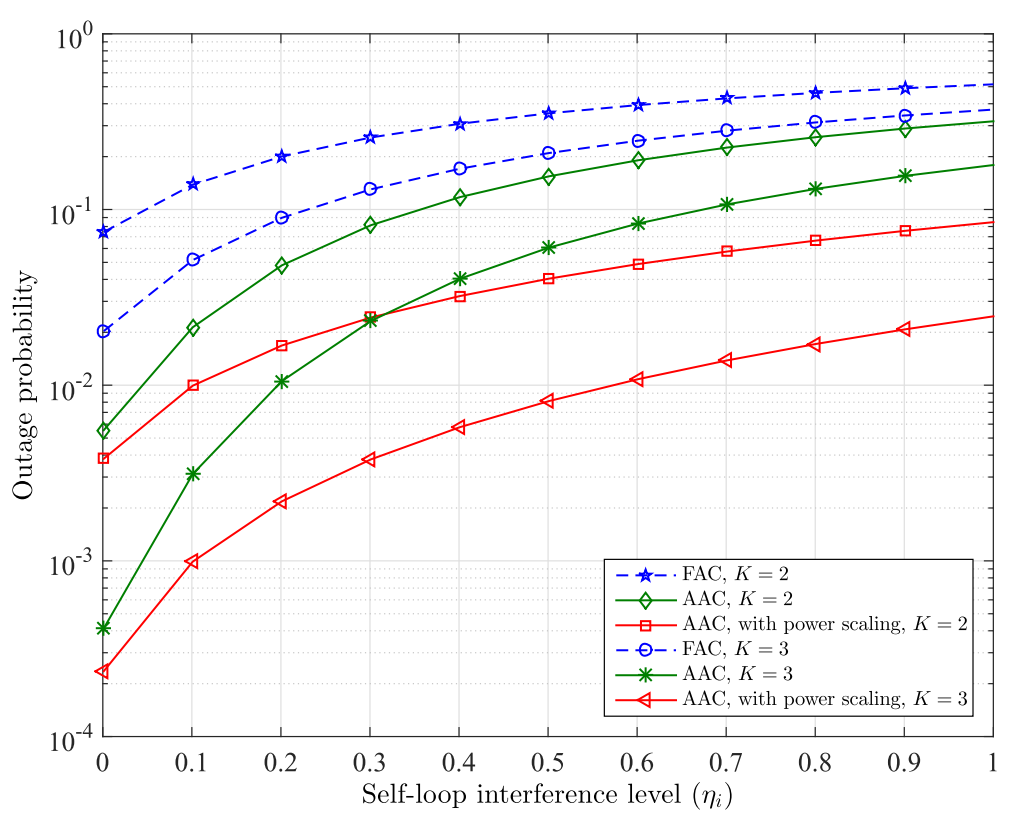

Fig. 8 Outage performance versus $\eta_{i}$ for different values of $K$ : FAC, AAC, and AAC with power scaling

Figure 7 compares outage performance between the cases of AAC and FAC versus the number of relays $K$ for two different SNR values $(0 \mathrm{~dB}, 5 \mathrm{~dB})$ at $\eta=0.01$. It can be seen that the outage performance improves when $K$ increases and the performance gain becomes larger when SNR increases. Therefore, in large-scale relay systems with a large number of relays, the outage probability quickly approaches zero. Moreover, the variation of $K$ on the outage performance is very small when SNR is very small and there is very little difference of outage performance between the two cases at small values of SNR.

Finally, Fig. 8 depicts the outage performance versus $\eta_{i}$ at $\mathrm{SNR}=10 \mathrm{~dB}$ for different values of $K$ under FAC, AAC without power scaling, and AAC with power scaling. We can see that when $\eta_{i}$ increases, the outage performance degrades. Also, the variation of $K$ has a strong effect on the outage performance in all cases.

\section{Conclusions}

This paper has considered wireless relay networks that employ $K$ full-duplex decode-and-forward relays to help a source to communicate with a destination. Joint relayantenna selection schemes are proposed and analyzed for two cases of antenna configurations, namely fixed antenna configuration (FAC) and adaptive antenna configuration (AAC). Closed-form expressions of the outage probability and average capacity were derived and provide important insights on the system performance. In particular, under FAC and without power scaling, the diversity order approaches $K$ as the self-interference level gets smaller, while it approaches zero whenever the SI level is nonzero and the SNR increases without bound. Under FAC and with power scaling, the diversity order approaches $K$ for any SI level. For the case of AAC and without power scaling, the diversity order approaches $2 K$ under small SI level. When power scaling is applied in AAC, the diversity order approaches $2 K$ at any SI level. All the analytical results are validated by computer simulations.

\section{Abbreviations}

AAC: Adaptive antenna configuration; AF: Amplify-and-forward; AGWN: Additive white Gaussian noise; DF: Decode-and-forward; FAC: Fixed antenna configuration; FD: Full-duplex; HD: Half-duplex; IOT: Internet of Things; NOMA: Non-orthogonal multiple-access; RAMS: Relay and Tx/Rx antenna mode selection scheme; RSI: Residual self-interference; Rx: Receive; SI:

Self-interference; SINR: Signal-to-interference-plus-noise ratio; Tx: Transmit; UAV: Unmanned aerial vehicle; 5G: Fifth generation

\section{Authors' contributions}

MSG carried out the main works of system model development, theoretical analysis and simulation, and writing of the manuscript. HHN contributed significantly to system model development, theoretical analysis and simulation, and writing of the manuscript. Both authors read and approved the final manuscript.

\section{Funding}

This research was supported by the Natural Sciences and Engineering Research Council (NSERC) of Canada. NSERC provides funding to cover expenses related to this work, but it does not participate in the design, analysis, and interpretation of data, and in writing the manuscript.

\section{Availability of data and materials}

Data sharing is available by emailing the first author (m_shirzadian@ut.ac.ir).

\section{Competing interests}

The authors declare that they have no competing interests. 


\section{Author details}

${ }^{1}$ Department of Electrical and Computer Engineering, University of Tehran, Tehran, Iran. ${ }^{2}$ Department of Electrical and Computer Engineering, University of Saskatchewan, Saskatoon, Canada.

Received: 7 August 2018 Accepted: 18 December 2019

Published online: 13 January 2020

\section{References}

1. Z. Zhang, K. Long, A. V. Vasilakos, L. Hanzo, Full-duplex wireless communications: Challenges, solutions, and future research directions. Proc. IEEE. 104(7), 1369-1409 (2016)

2. Z. Zhang, X. Chai, K. Long, A. Vasilakos, L. Hanzo, Full duplex techniques for $5 \mathrm{G}$ networks: Self-interference cancellation, protocol design, and relay selection. IEEE Commun. Mag. 53(5), 128-137 (2015)

3. L. Li, H. Poor, L. Hanzo, Non-coherent successive relaying and cooperation: Principles, designs, and applications. IEEE Commun. Surv. Tut. 17(3), 1708-1737 (2015)

4. A. Sabharwal, P. Schniter, D. Guo, D. W. Bliss, S. Rangarajan, R. Wichman, In-band full-duplex wireless: Challenges and opportunities. IEEE J. Sel. Areas Commun. 32(9), 1637-1652 (2014)

5. T. Riihonen, S. Werner, R. Wichman, Mitigation of loopback selfinterference in full-duplex MIMO relays. IEEE Trans. Sig. Process. 59(12), 5983-5993 (2011)

6. E. Everett, M. Duarte, C. Dick, A. Sabharwal, in Proc. Asilomar Conf. Signals Syst. Comp. Empowering full-duplex wireless communication by exploiting directional diversity, (2011), pp. 2002-2006. https://doi.org/10. 1109/acssc.2011.6190376

7. H. Jin, V. Leung, Performance analysis of full-duplex relaying employing fiber-connected distributed antennas. IEEE Trans. Veh. Technol. 61(1), 146-160 (2013)

8. T. Kim, A. Paulraj, in IEEE WCNC. Outage probability of amplify-and-forward cooperation with full duplex relay (IEEE, 2012), pp. 75-79. https://doi.org/ 10.1109/wenc.2012.6214473

9. T. K. Baranwal, D. S. Michalopoulos, R. Schober, Outage analysis of multihop full duplex relaying. IEEE Commun. Lett. 11(1), 63-66 (2013)

10. T. Riihonen, S. Werner, R. Wichman, Hybrid full-duplex/half duplex relaying with transmit power adaptation. IEEE Trans. Wireless Commun. 10(9), 3074-3085 (2011)

11. I. Krikidis, H. Suraweera, S. Yang, K. Berberidis, Full-duplex relaying over block fading channel: A diversity perspective. IEEE Trans. Wirel. Commun. 11(12), 4524-4535 (2012)

12. S. Li, K. Yang, M. Zhou, J. Wu, L. Song, Y. Li, H. Li, Full-duplex amplify-and-forward relaying: Power and location optimization. IEEE Trans. Veh. Technol. 66(9), 8458-8468 (2017)

13. A. Almradi, A. K. Hamdi, MIMO full-duplex relaying in the presence of co-channel interference. IEEE Trans. Veh. Technol. 66(6), 4874-4885 (2017)

14. H. A. Suraweera, I. Krikidis, C. Yuen, in Proc. IEEE ICC. Antenna selection in the full-duplex multi-antenna relay channel, (2013), pp. 4823-4828. https://doi.org/10.1109/icc.2013.6655338

15. H. Suraweera, I. Krikidis, G. Zheng, C. Yuen, P. J. Smith, Low-complexity end-to-end performance optimization in MIMO full-duplex relay systems. IEEE Trans. Wirel. Commun. 13(2), 913-927 (2014)

16. B. Ji, Y. Li, Y. Meng, Y. Wang, K. Song, C. Han, H. Wen, L. Song, Performance analysis of two-way full-duplex relay with antenna selection under Nakagami channels. EURASIP J. Wirel. Commun. Netw. 2018 (2018). https://doi.org/10.1186/s13638-018-1283-2

17. H. Cui, M. Ma, L. Song, B. Jiao, Relay selection for two-way full duplex relay networks with amplify-and-forward protocol. IEEE Trans. Wirel. Commun. 13(7), 3768-3777 (2014)

18. K. Yang, H. Cui, L. Song, Y. Li, Efficient full-duplex relaying with joint antenna-relay selection and self-interference suppression. IEEE Trans. Wirel. Commun. 14(7), 3991-4005 (2015)

19. Y. Wang, Y. Xu, N. Li, W. Xie, K. Xu, X. Xia, Relay selection of full-duplex decode-and forward relaying over Nakagami-m fading channels. IET Commun. 10(2), 170-179 (2016)

20. Q. Li, S. Feng, X. Ge, G. Mao, L. Hanzo, On the performance of full-duplex multi-relay channels with DF relays. IEEE Trans. Veh. Technol. 66(10), 9550-9554 (2017)
21. T. A. Le, H. Y. Kong, Energy harvesting relay-antenna selection in cooperative MIMO/NOMA network over Rayleigh fading. Wirel. Netw., $1-13(2019)$

22. K. Song, B. Ji, C. Li, L. Yang, Outage analysis for simultaneous wireless information and power transfer in dual-hop relaying networks. Wirel. Netw. 25(2), 837-844 (2019)

23. B. Ji, Y. Li, B. Zhou, C. Li, K. Song, H. Wen, Performance analysis of UAV relay assisted loT communication network enhanced with energy harvesting. IEEE Access. 7, 38738-38747 (2019). https://doi.org/10.1109/ACCESS. 2019.2906088

24. R. Narasimhan, A. Ekbal, J. M. Cioffi, in IEEE ICC. Finite-SNR diversitymultiplexing tradeoff of space-time codes (IEEE, 2005), pp. 458-462. https://doi.org/10.1109/icc.2005.1494394

25. I. S. Gradshteyn, I. M. Ryzhik, Table of Integrals, Series, and Products, 8th ed. (Academic, New York, 2014)

\section{Publisher's Note}

Springer Nature remains neutral with regard to jurisdictional claims in published maps and institutional affiliations.

\section{Submit your manuscript to a SpringerOpen ${ }^{\circ}$ journal and benefit from:}

- Convenient online submission

Rigorous peer review

- Open access: articles freely available online

- High visibility within the field

- Retaining the copyright to your article

Submit your next manuscript at $>$ springeropen.com 